\title{
Review Article \\ Epithelial to Mesenchymal Transition in a Clinical Perspective
}

\author{
Jennifer Pasquier, ${ }^{1,2}$ Nadine Abu-Kaoud, ${ }^{1}$ Haya Al Thani, ${ }^{1,2}$ and Arash Rafii ${ }^{1,2}$ \\ ${ }^{1}$ Stem Cell and Microenvironment Laboratory, Department of Genetic Medicine and Obstetrics and Gynecology, \\ Weill Cornell Medical College in Qatar, Education City, Qatar Foundation, P.O. Box 24144, Doha, Qatar \\ ${ }^{2}$ Department of Genetic Medicine, Weill Cornell Medical College, New York, NY 10021, USA
}

Correspondence should be addressed to Jennifer Pasquier; jep2026@qatar-med.cornell.edu and Arash Rafii; jat2021@qatar-med.cornell.edu

Received 24 September 2014; Accepted 13 January 2015

Academic Editor: Sandra Cascio

Copyright (C) 2015 Jennifer Pasquier et al. This is an open access article distributed under the Creative Commons Attribution License, which permits unrestricted use, distribution, and reproduction in any medium, provided the original work is properly cited.

\begin{abstract}
Tumor growth and metastatic dissemination rely on cellular plasticity. Among the different phenotypes acquired by cancer cells, epithelial to mesenchymal transition (EMT) has been extensively illustrated. Indeed, this transition allows an epithelial polarized cell to acquire a more mesenchymal phenotype with increased mobility and invasiveness. The role of EMT is quite clear during developmental stage. In the neoplastic context in many tumors EMT has been associated with a more aggressive tumor phenotype including local invasion and distant metastasis. EMT allows the cell to invade surrounding tissues and survive in the general circulation and through a stem cell phenotype grown in the host organ. The molecular pathways underlying EMT have also been clearly defined and their description is beyond the scope of this review. Here we will summarize and analyze the attempts made to block EMT in the therapeutic context. Indeed, till today, most of the studies are made in animal models. Few clinical trials are ongoing with no obvious benefits of EMT inhibitors yet. We point out the limitations of EMT targeting such tumor heterogeneity or the dynamics of EMT during disease progression.
\end{abstract}

\section{Introduction}

Despite the improvement of treatment regimens, cancer remains a leading cause of death worldwide. Metastatic disease is responsible for the majority of cancer-induced mortality [1]. The development of new therapeutic strategies targeting key factors driving metastasis remains a challenging goal for both clinicians and scientists. Metastasis is artificially divided into a series of sequential highly organized and organ specific steps [2]. Among these steps is the acquisition of migratory and invasive proprieties by cancer cells, which can be achieved through epithelial-mesenchymal transition (EMT) [3-6].

First described in embryogenesis, EMT is a cellular reprogramming process in which epithelial cells acquire a mesenchymal phenotype [7]. During this transformation, epithelial cells lose their polygonal shape and ability to grow in colonies, but they acquire spindle-shaped morphology and exhibit a more motile and invasive behavior [8]. These phenotypic changes are associated with proteins and gene modifications in different interconnected families such as transcription factors, cadherins, catenins, matrix metalloproteases (MMPs), or growth receptors $[9,10]$.

While EMT has been well accepted and demonstrated in vivo during embryogenesis, its implication in the metastatic process is still debated [11-16]. Identifying the EMT process in neoplastic disease is difficult since cells undergoing EMT share many molecular and morphological characteristics with the surrounding stromal fibroblasts. Moreover, although primary carcinoma or circulating tumor cells (CTCs) display EMT features, cells present in the distant metastases site are generally epithelial [17]. In 2002, Their proposed an explanation to such observation by describing the reversible EMT metastasis model in which primary epithelial tumor cells activate EMT to invade distant sites, and, upon arriving, they undergo a MET (mesenchymal-epithelial transition) to form an epithelial metastatic lesion [18].

Numerous reviews have comprehensively described EMT in cancer as well as the molecular pathways implicated in EMT or MET $[17,19-21]$. The description of such findings 
is beyond the scope of this review. Here, we focus on the latest research on EMT in the clinical context for prognostic or therapeutic or strategies.

\section{Can We Use EMT to Predict Patient's Outcome?}

Recently, the detection of circulating tumor cells above a defined cut-off has been associated with poor prognosis in different cancers such as breast or prostate tumors [22, 23]. Circulating tumor cells, as well as metastatic lesions, of many different cancers present EMT characteristic [24-30]. Many studies investigated whether the expression of EMT markers would be associated with poor patient prognosis. The aberrant expression of Snail is related to poor patient survival in breast [31-34], ovarian [33, 35, 36], hepatocellular [37$40]$, and colorectal carcinomas [41, 42]. Twist overexpression is associated with a poor clinical outcome in many cancers such as bladder cancer [43], breast cancer [34], oral squamous cell carcinoma [44], ovarian cancer $[45,46]$, or cervical cancer [47]. Vimentin overexpression in cancers and its correlation with growth and metastasis suggest that it might be an indicator of poor prognostic for many cancers [48]. In bladder cancer, a study of eleven different cell lines revealed that the loss of E-cadherin expression is a marker of poor response to the monoclonal antibody cetuximab, which blocks EGFR binding [49]. More recently, Twist-1 promoter hypermethylation, studied on 65 surgically resected specimens, was shown to be a useful molecular marker for predicting prognosis and contralateral cervical lymph node metastases in patients with tonsillar squamous cell carcinoma [50].

The increasing amount of data on single EMT indicators urged the investigation of the correlation between several markers on patients' prognosis. A 4-EMT genes signature (E-cadherin (CDH1), inhibitor of DNA binding 2 (ID2), matrix metalloproteinase 9 (MMP9), and transcription factor 3 (TCF3)) was used to predict clinical outcome in a cohort of 128 hepatocellular carcinoma patients and then validated in an independent cohort of 231 patients with hepatocellular carcinoma from three different institutions [51]. The authors claimed that this 4-gene signature could improve patients' survival prediction on the risk score and tumor stage. Recently, in a study including surgical specimens from 78 cases of esophageal squamous cell carcinoma resected without preoperative treatment between 2001 and 2013, Niwa et al. demonstrated that the vimentin/E-cadherin ratio was correlated with tumor invasion and can serve as an independent prognostic factor among chemonaive patients [52]. In an analysis of 100 surgically resected hepatic tumors, EMT markers Twist-1 and Zeb-2 were shown to be involved in early disease recurrence in hepatocellular carcinoma and served as good prognosis markers [53]. In a different study on paraffinembedded hepatocellular carcinoma tissues $(n=113)$ and their corresponding peritumoral normal tissues $(n=106)$, although the expression of 3-EMT-related proteins, S100A4, vimentin, and E-cadherin was studied, the authors reported that E-cadherin alone can be used as a direct prognosticator of negative outcome [54]. The prognostic significance of Ecadherin, twist, and vimentin was assayed in 121 patients with bladder cancer and, in this study, only vimentin appears as an independent predictor for cancer progression and survival [55].

Cancer stem cells have emerged as a particular entity within tumor cell plasticity. They have the ability to reinitiate the tumor in serial engraftment assays; they are more resistant to treatment than the bulk of the tumor and their role in the occurrence of metastasis has been suggested in several studies [1,56-58]. Their identification relies on functional proprieties (spheroid formation in 3D media, asymmetric division, and serial passages in NOD/SCID mice) and specific markers. Several authors have described an increase of tumor stemness when cancer cells undergo EMT, leading to the study of stem cell associated markers combined with EMTrelated markers as prognosis indicators. Luo et al. studied the correlation of SOX2, OCT4, and Nanog with E-cadherin, Ncadherin, and Snail in a nasopharyngeal carcinoma cohort of 122 patients [59]. They demonstrated that OCT4 and Nanog could be used as poor prognosis factor and are linked with the progression of the invasive front. In two different clinical studies, one on 119 human cholangiocarcinoma patients [60] and one on 276 consecutive primary gastric cancers and 54 matched lymph node metastases [61], the same team revealed that EMT markers (Snail-1, Zeb-1, E-cadherin, vimentin, and beta-catenin) and the CSC marker, CD44, are strongly correlated. Moreover, they revealed that the simultaneous expression of Snail-1, vimentin, E-cadherin, and CD44 was associated with advanced stage, metastasis, and invasion and was an independent indicator for disease-free survival.

Accumulating evidences in the literature are reflecting the regulatory roles of microRNAs (miRNAs) on EMT phenotype [62]. miRNAs can be optimal markers of specific disease or a patient' prognosis. Indeed, they can be found and quantified in many different biological fluids, including blood, urine, and cerebrospinal fluid; they also display great stability (even after boiling, freeze-thaw cycles, or low or high $\mathrm{pH}$ conditions). For instance, tumors with low expression of miR-335 and miR-126, 2 miRNA known to inhibit the first step of EMT, have been reported to present more probability to develop metastasis than tumors with higher expression of these miRNA [63]. In the blood, qPCR analysis of miR10b, miR-34, and miR-155 allows the discrimination between patients with breast cancer metastasis and healthy controls. These miRNA play an important role in regulating EMT in response to TGF $\beta$. A meta-analysis of 17 studies with various carcinomas uncovered the role of miR-21 (an oncomiR known to promote EMT through TGF $\beta$ pathway) as a poor prognosis biomarker in breast, squamous cell carcinoma, astrocytoma, and gastric cancer [64].

As described above, many EMT markers or their derivatives have been associated with patients' prognosis in different studies. However, while an EMT phenotype seems clearly associated with an increased metastatic phenotype, the use of such markers has not yet been translated into clinical practice. The requirements for an assay to be usable in clinical practice are quite stringent. Indeed, any prognosis marker has to first 
display great robustness. It should be reproducible among different laboratories and between pathologists. As prognostic markers will be used to give adjuvant therapy, their specificity should be quite high allowing the identification of patients who would not benefit from adjuvant treatment. While EMT markers have been independently associated with patients' prognosis, they have not yet been used in clinical practice for several reasons.

Tumor heterogeneity is one of the main reasons; the expression of EMT markers can vary at different locations of a tumor (usually increased mesenchymal phenotype at the periphery). Clear cut-offs are critical; most of the markers used in classical pathology have clear cut-offs (mitosis per field, Her2 overexpression, etc.). For EMT markers, defining the cut-offs might be difficult as different tumors display different levels of epithelial or mesenchymal phenotype and it is hard to clearly attribute a value for a particular marker. These are few difficulties among others including technical issues to do multiplex assays and the lack of large multicentric prospective trials.

One solution might be the advent of oncogenomic prognosis assays based on gene expression [65]. Currently, more than 100 clinical trials in cancer disease are ongoing using EMT as a keyword. Most of them study the prevalence of EMT markers in different cancers and their potential use as prognosis factor. The data from these studies will help us determine the clinical context where EMT could be used to tailor patients' treatment.

\section{Can We Use EMT Effectors to Treat Patients?}

EMT is an extremely well-organized process, activated in response to a combination of extracellular cues from the tumor microenvironment. EMT-inducing signals seem to be cell or tissue specific and require the cooperation between multiple signaling pathways and regulators. We considered the potential targets in three different groups classified based on their role during EMT: the molecular effectors executing EMT, the transcription factors acting as regulators to orchestrate EMT, and extracellular inducers that engage the cells in EMT.

3.1. Effectors. EMT effectors are mostly proteins that define the epithelial or mesenchymal phenotype of a cell. A key feature of EMT is the switch from E-cadherin (marker of epithelial cells) to N-cadherin (makers of mesenchymal cells) [9]. Targeting these cadherins in order to avoid a loss of Ecadherin or an upregulation of N-cadherin could therefore be a promising strategy.

Several groups studied the transfection of E-cadherin in highly mesenchymal and invasive cells and showed a reversion of the poorly differentiated carcinoma into a welldifferentiated one with a minimally invasive epithelial phenotype [66-70]. In breast cancer, it has been demonstrated that salinomycin can selectively kill E-cadherin-negative breast epithelial cells as compared with E-cadherin-positive cells in NOD/SCID and Balb/c mice model [71]. Global gene expression analyses of breast tissues isolated directly from patients display that salinomycin treatment results in the loss of cancer stem cell (CSC) expression. The tumor suppressor role of E-cadherin has been established in many cancers including hepatocellular carcinoma [72], esophagus [73], melanoma [74], breast cancer [75, 76], or squamous cell carcinoma of the skin, head, and neck $[77,78]$. However, in ovarian tumors, E-cadherin is consistently upregulated and maintained in ovarian carcinoma cells that metastasize to the peritoneum and omentum [79]. E-cadherin expression has been found in patients with a family history of ovarian cancer, proposing a potential role of E-cadherin in tumor initiation and/or progression in this particular cancer [80]. Concordantly, several recent studies point to a promoting role of E-cadherin during tumor progression in different epithelial cancers such as ovarian, breast, or brain cancer (reviewed in [81]). An epithelial phenotype has been also correlated with an increase in cancer stemness and engraftment in host organs in prostate cancer [82]. Overall, targeting Ecadherin seems to be difficult due to its ambiguous role in carcinogenesis.

Inhibition of $\mathrm{N}$-cadherin has been assessed in several studies [83, 84]. Shintani et al. reported that, in a mouse model of pancreatic cancer, the peptide ADH-1 is able to block N-cadherin and prevent tumor progression [85]. In head and neck cancer cell line, quercetin has been shown to significantly reduce the migration ability of sphere cells by decreasing N-cadherin production [86]. Recently, Sadler et al. demonstrated that targeting $\mathrm{N}$-cadherin using a neutralizing antibody may be a good therapeutic strategy to treat multiple myeloma [87].

Vimentin is also a canonical marker of mesenchymal phenotype and therefore an important effector of EMT [48]. Few reports have shown a direct inhibition of vimentin. Lahat and collaborator suggested that the withaferin-A induces vimentin degradation in a panel of soft tissue sarcoma xenograft experiments, leading to the inhibition of growth, local recurrence, and metastasis [88]. In prostate tumors, both silibinin and flavonolignan inhibited invasion, motility, and migration of the cancer cells via downregulation of vimentin in cancer cell lines and mice models $[89,90]$. Finally, salinomycin, an antibiotic, reduced significantly vimentin level and induced increase in E-cadherin expression in $\mathrm{CD}_{133^{+}}$colorectal cancer cell lines HT29 and SW480 resulting in decreased malignant traits [91].

None of these strategies are currently tested in a clinical context. Indeed, EMT effectors have a complex role and their function might be time and context dependent during the metastatic process such as illustrated by the dual role of Ecadherin. Some additional key EMT effector molecules are proteins that promote cell migration and invasion during the process such as fibronectin, PDGF/PDGF receptor autocrine loop, $\mathrm{Cd} 44$, or integrin $\beta 6$ [17]. Hence, these proteins might also be considered as potential targets to counter the EMT process.

3.2. Regulators. EMT regulators are a core of transcription factor such as Snail-1/Snail-1, basic helix-loop-helix family (E47, E2-2, and Twist-1/Twist-2), and Zeb-1/Zeb-1 [21, 92]. The role of these transcription factors in proliferation, 
invasion, and migration of epithelial tumors has been well described and their use as a target to block EMT process seems appealing [41, 93-95].

da Silva et al. reported that the inhibition of Twist-1 in metastatic oral squamous cell carcinoma (OSCC) induced a potent inhibition of cell invasiveness in vitro as well as in vivo using an orthotropic mouse model of metastatic OSCC [44]. The secreted frizzled-related protein (sFRP1 and sFRP2) two Wnt antagonists enhance the expression of Ecadherin through the inhibition of Twist- 1 and suppress the invasiveness of cervical cancer in vivo in a xenograft animal model [96]. The bone morphogenetic protein 7 (BMP7) is a potential metastasis inhibitor that disrupts EMT through Twist-1 inhibition in melanoma WM-266-4 and HEK293T cell lines [97]. Arumugam et al. have shown using many different pancreatic cell lines that silencing Zeb-1 not only restored the expression of epithelial marker genes, but also increased cellular sensitivity to therapeutic reagents [98]. In a lung carcinoma cell line, the knockdown of Snail or Twist-1 is able to restore the cell chemosensitivity to cisplatin $[99,100]$.

The sulforaphane, an organosulfur compound, is able to downregulate Twist-1 as well as other EMT proteins like vimentin leading to a decrease of stemness properties in PANC-1, MIA PaCa-2, AsPC-1, and Bx PC-3 pancreatic cells lines [101]. Recently, moscatilin was shown to target the Akt-Twist-1 dependent pathway and decrease the migration and metastasis of MDA-MB-231 breast cancer cell line [102]. Fucoidan was also described to inhibit EMT in breast cancer cell lines such as $4 \mathrm{~T} 1$ and MDA-MB-231 through the decreased Twist-1, Snail, and Slug expression [103]. In 2014, Myung and collaborators demonstrated that the knockdown of Snail with siRNA technique in three glioblastoma cell lines (KNS42, U87, and U373) suppresses the proliferation, viability, migration, and invasion of cells by disrupting the EMT process [104].

Despite the promising results in preclinical studies, overall, EMT core transcription factors remain technically challenging to target in a clinical setting. However, a clinical trial is currently investigating the molecular mechanism and clinical significance of the interplay between Twist-1 and other EMT regulators through microRNA-29 family in head and neck squamous cell carcinoma (NCT01927354).

3.3. Inducers. The principal inducers of EMT are proteins from the TGF $\beta$ (TGF $\beta 1$, TGF $\beta 2$, TGF $\beta 3$, inhibins, activin, anti-Müllerian hormone, bone morphogenetic protein, decapentaplegic, and Vg-1) and the growth factor (fgf, hgf, egf, and igf1) families [21]. High-throughput drug screening has been performed to identify potential inhibitors of EMT in response to various inducers. The majority of molecules selected inhibit specific EMT-inducing signals used in the screen. For instance, rapamycin and 17-AGG have been shown to inhibit TGF $\beta$-induced EMT through the modification of TGF $\beta$ pathway itself as assessed by a global gene expression profile from a cell culture model of TGF $\beta$-induced EMT [105]. Inhibitors of ALK5, MEK, and SRC are able to block EMT in response to EGF, HGF, and IGF$1[105,106]$. In 2011, two different groups proposed c-MET as a potential therapeutic target in hepatocellular carcinoma cell lines Huh7, Hep3B, MHCC97-L, and MHCC97-H [107] and BNL CL.2 (BNL) and BNL 1ME A. 7R.1 (1MEA) [108]. In prostate cancer, inhibition of c-Met expression and Metmediated signaling by Frzb leads to the upregulation of epithelial markers and a decrease of the mesenchymal traits in a xenograft mouse model [109].

The most studied inducer of EMT remains TGF $\beta$ [110112]. Targeting TGF $\beta$ pathway to alter EMT induced tumor cell invasion may be appropriate as metastasis prevention strategies in early stage carcinomas. SD-093 and LY-580276, two competitive inhibitors for the ATP-binding site of TGF $\beta$ RI kinase, disrupt EMT and tumor cell migration in many cancers [113, 114]. EW-7203, EW-7195, and EW-7197, specific TGF $\beta$ /ALK5 inhibitors available as orally administered drugs $[115,116]$, have been shown to inhibit EMT in both TGF $\beta$ treated breast cancer cells and $4 \mathrm{~T} 1$ orthotropic xenograft mice [117]. Bone morphogenetic protein 7 (BMP7) was revealed as a potential inhibitor of EMT induced by TGF $\beta$ in thirty liver tissue samples of patients with cholangiocarcinoma [118].

Currently, the only compounds interfering with EMT in clinical trial are the ones able to block EMT inducers. In 2008, already, the LY2157299, a clinical selective TGF $\beta 1$ receptor inhibitor, was undergoing a still unpublished phase I trial for colon, prostate, and adrenocortical or breast cancer and malignant melanoma patients [119]. That same year, another preclinical trial using human xenografts Calu6 (nonsmall-cell lung cancer) and MX1 (breast cancer) implanted subcutaneously in nude mice demonstrated that LY2157299 is able to reduce the tumor growth [120]. LY2157299 is now known to display antitumor effects in patients with glioblastoma and hepatocellular carcinoma [121]. LY2157299 is currently tested in four clinical trials, in patients recruiting state: Phase $\mathrm{Ib} / \mathrm{II}$ in stages II-IV pancreatic cancer of LY2157299 combined with gemcitabine versus gemcitabine plus placebo (NCT01373164); Phase II in HCC patients with disease progression on sorafenib or who are not eligible to receive sorafenib (NCT01246986); Phase Ib/IIa study combining LY2157299 with standard temozolomide based radiochemotherapy in patients with newly diagnosed malignant glioma (NCT01220271); and Phase II Study of LY2157299 monotherapy or LY2157299 plus Lomustine therapy compared to Lomustine monotherapy in patients with recurrent glioblastoma (NCT01582269).

Erlotinib, an EGF receptor tyrosine kinase inhibitor, is approved for the treatment of second- and third-line advanced non-small-cell lung cancers $[122,123]$. Interestingly, its efficacy is correlated with the EMT status of the cells; higher E-cadherin levels indicate sensitivity, whereas higher vimentin and Zeb-1 levels indicate resistance. Thus, in 2012, a randomized phase II trial on 132 patients with non-smallcell lung cancer evaluated the effect of erlotinib combined with the isoform selective histone deacetylase inhibitors, entinostat, known to prevent the resistance by reverting the cancer cell mesenchymal phenotype to an epithelial one [124]. Even if entinostat failed at improving the outcome of patients, the study revealed that E-cadherin expression levels at time of diagnosis could portray the sensitivity to 


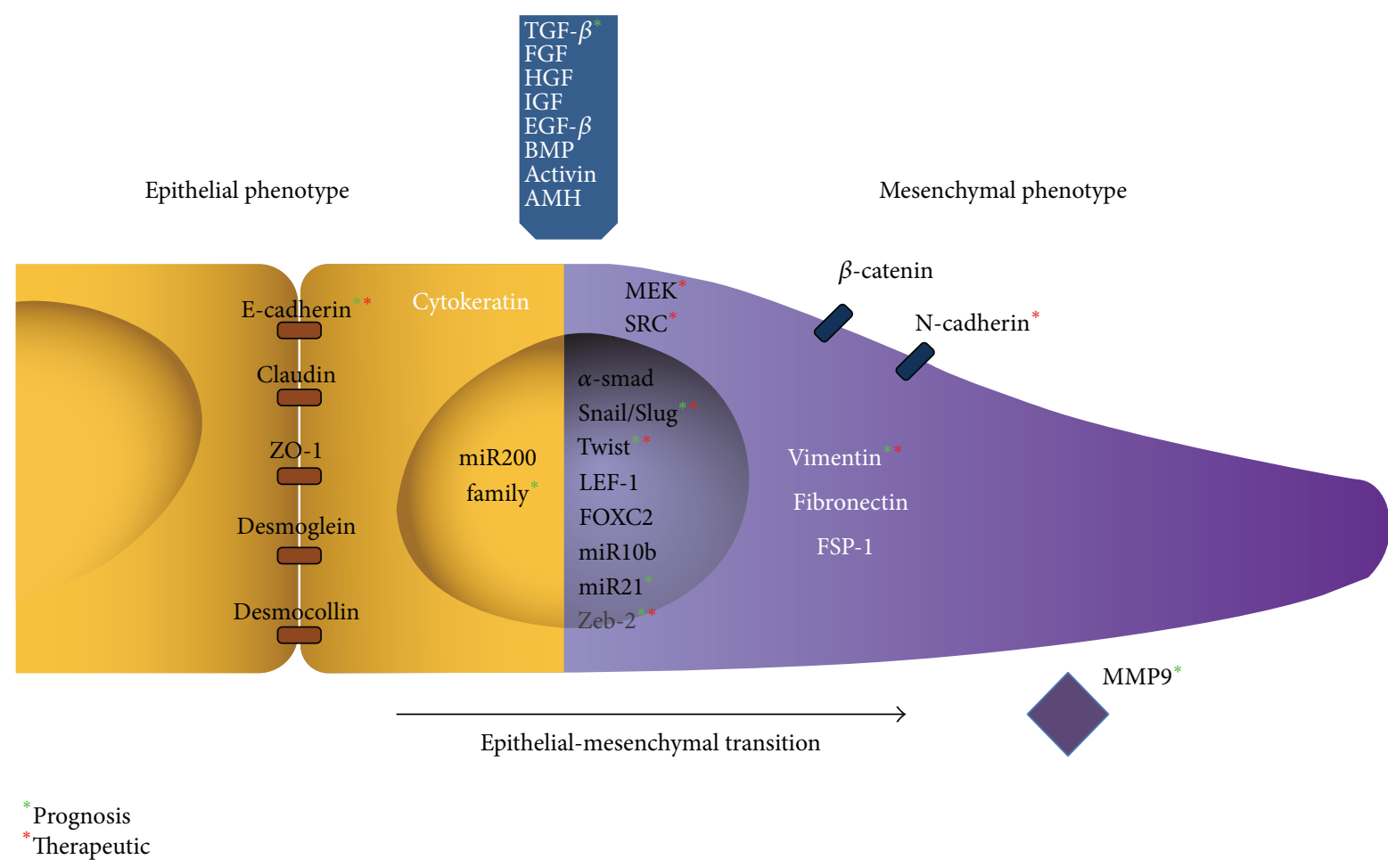

FIGURE 1: Epithelial to mesenchymal transition with effectors and inducers studied in the prognostic or therapeutic context. Green asterisk: implicated in prognosis. Red asterisk: targeted therapeutically.

HDACi/EGFR-TKI inhibition providing the basis for a biomarker-driven validation.

In 2014, the GC1008 (fresolimumab), a human anti-TGF $\beta$ monoclonal antibody, has undergone a phase I clinical trial in patients with advanced malignant melanoma or renal cell carcinoma [125]. 29 patients, 28 with malignant melanoma and 1 with renal cell carcinoma, were included and received intravenous GC1008 at $0.1,0.3,1,3,10$, or $15 \mathrm{mg} / \mathrm{kg}$ on days $0,28,42$, and 56 . It demonstrated that GC1008 was presenting an acceptable safety and toxicity, and the maximum dose, $15 \mathrm{mg} / \mathrm{kg}$, was determined. Multiple doses of GC1008 demonstrated only preliminary evidence of antitumor activity, allowing further studies of single agent and combination treatments in clinical trials. In fact, GC-1008 is currently tested in two clinical studies: fresolimumab and radiotherapy in metastatic breast cancer (NCT01401062) and safety and imaging study of GC1008 in glioma (NCT01472731).

\section{A Moving Target}

Despite the massive amount of preclinical data in most cancer types, there is still no clear cancer treatment specifically targeting EMT. Many aspects of tumor biology can explain the gap between the preclinical and clinical data.

In most preclinical studies an induced model of EMT is used to demonstrate the implication of EMT in tumor spread and then to demonstrate the efficacy of targeting EMT in achieving tumor response. These models are usually quite far from clinical reality. They do not perfectly reproduce tumor heterogeneity which is increased at the metastatic stage as demonstrated by several studies of tumor phylogeny [126] Such heterogeneity can be the result of cell intrinsic genomic differences or interaction with the microenvironment [127133]. Tumor progression acquired through EMT can be transient and represent only a small timeframe in a patient's disease. Hence, targeting EMT will not have similar effect as in preclinical studies where EMT is constitutively activated. Many of the factors have dual or ambivalent roles. The recent demonstration of the role of MET in the establishment of metastasis in the host organ and its relationship to stemness in specific tumors adds a new degree of complexity to antiEMT strategies. One could somehow potentially increase cancer stemness and improve host organ homing by inhibiting EMT.

\section{Conclusion}

Overall, despite the tremendous amount of preclinical data on the implication of EMT in cancer progression, there is still no routine clinical translation at both prognosis and therapeutic levels (Figure 1). Here, while we point out the different elements of the EMT cascades that could be targeted, we also underline the difficulties to translate the preclinical findings in routine clinic. However, we can hypothesize that as we enter the era of precision and personalized medicine, new technologies (next gene sequencing, circulating tumor cells, circulating tumor DNA, etc.) will help us better define patients' specific disease at precise time points during disease evolution. Such studies might then really illustrate whether EMT has a role in neoplastic evolution and point out 
the appropriate therapeutic window where EMT inhibition could lead to improved survival in patients.

\section{Conflict of Interests}

The authors declare that there is no conflict of interests regarding the publication of this paper.

\section{Acknowledgment}

The authors would like to acknowledge the Chalhoub Group for their support of cancer research.

\section{References}

[1] C. L. Chaffer and R. A. Weinberg, "A perspective on cancer cell metastasis," Science, vol. 331, no. 6024, pp. 1559-1564, 2011.

[2] T. J. Yeatman and G. L. Nicolson, "Molecular basis of tumor progression: mechanisms of organ-specific tumor metastasis," Seminars in Surgical Oncology, vol. 9, no. 3, pp. 256-263, 1993.

[3] R. Kalluri and R. A. Weinberg, "The basics of epithelialmesenchymal transition," The Journal of Clinical Investigation, vol. 119, no. 6, pp. 1420-1428, 2009.

[4] K. Polyak and R. A. Weinberg, "Transitions between epithelial and mesenchymal states: acquisition of malignant and stem cell traits," Nature Reviews Cancer, vol. 9, no. 4, pp. 265-273, 2009.

[5] J. P. Thiery, H. Acloque, R. Y. J. Huang, and M. A. Nieto, "Epithelial-mesenchymal transitions in development and disease," Cell, vol. 139, no. 5, pp. 871-890, 2009.

[6] L. Wan, K. Pantel, and Y. Kang, "Tumor metastasis: moving new biological insights into the clinic," Nature Medicine, vol. 19, no. 11, pp. 1450-1464, 2013.

[7] D. C. Radisky, "Epithellial-mesenchymal transition," Journal of Cell Science, vol. 118, no. 19, pp. 4325-4326, 2005.

[8] J. P. Thiery and J. P. Sleeman, "Complex networks orchestrate epithelial-mesenchymal transitions," Nature Reviews Molecular Cell Biology, vol. 7, no. 2, pp. 131-142, 2006.

[9] U. Cavallaro and G. Christofori, "Cell adhesion and signalling by cadherins and Ig-CAMs in cancer," Nature Reviews Cancer, vol. 4, no. 2, pp. 118-132, 2004.

[10] B. M. Gumbiner, "Regulation of cadherin-mediated adhesion in morphogenesis," Nature Reviews Molecular Cell Biology, vol. 6, no. 8, pp. 622-634, 2005.

[11] M. H. Chui, "Insights into cancer metastasis from a clinicopathologic perspective: Epithelial-Mesenchymal Transition is not a necessary step," International Journal of Cancer, vol. 132, no. 7, pp. 1487-1495, 2013.

[12] K. Garber, "Epithelial-to-mesenchymal transition is important to metastasis, but questions remain," Journal of the National Cancer Institute, vol. 100, no. 4, pp. 232-239, 2008.

[13] H. Ledford, "Cancer theory faces doubts," Nature, vol. 472, no. 7343, article 273, 2011.

[14] D. Tarin, E. W. Thompson, and D. F. Newgreen, "The fallacy of epithelial mesenchymal transition in neoplasia," Cancer Research, vol. 65, no. 14, pp. 5996-6000, 2005.

[15] S. Thomson, E. Buck, F. Petti et al., "Epithelial to mesenchymal transition is a determinant of sensitivity of non-small-cell lung carcinoma cell lines and xenografts to epidermal growth factor receptor inhibition," Cancer Research, vol. 65, no. 20, pp. 94559462, 2005.
[16] J. Zavadil, J. Haley, R. Kalluri, S. K. Muthuswamy, and E. Thompson, "Epithelial-mesenchymal transition," Cancer Research, vol. 68, no. 23, pp. 9574-9577, 2008.

[17] J. H. Tsai and J. Yang, "Epithelial-mesenchymal plasticity in carcinoma metastasis," Genes and Development, vol. 27, no. 20, pp. 2192-2206, 2013.

[18] J. P. Their, "Epithelial-mesenchymal transitions in tumor progression," Nature Reviews Cancer, vol. 2, no. 6, pp. 442-454, 2002.

[19] B. D. Craene and G. Berx, "Regulatory networks defining EMT during cancer initiation and progression," Nature Reviews Cancer, vol. 13, no. 2, pp. 97-110, 2013.

[20] H. Zheng and Y. Kang, "Multilayer control of the EMT master regulators," Oncogene, vol. 33, no. 14, pp. 1755-1763, 2014.

[21] S. Lamouille, J. Xu, and R. Derynck, "Molecular mechanisms of epithelial-mesenchymal transition," Nature Reviews Molecular Cell Biology, vol. 15, no. 3, pp. 178-196, 2014.

[22] C. Alix-Panabières, H. Schwarzenbach, and K. Pantel, "Circulating tumor cells and circulating tumor DNA," Annual Review of Medicine, vol. 63, pp. 199-215, 2012.

[23] A. Rafii, F. Vidal, G. Rathat, and C. Alix-Panabières, "Circulating tumor cells: cornerstone of personalized medicine," Journal de Gynécologie Obstétrique et Biologie de la Reproduction, vol. 43, no. 9, pp. 640-648, 2014.

[24] A. Bonnomet, L. Syne, A. Brysse et al., "A dynamic in vivo model of epithelial-to-mesenchymal transitions in circulating tumor cells and metastases of breast cancer," Oncogene, vol. 31, no. 33, pp. 3741-3753, 2012.

[25] N. P. A. D. Gunasinghe, A. Wells, E. W. Thompson, and H. J. Hugo, "Mesenchymal-epithelial transition (MET) as a mechanism for metastatic colonisation in breast cancer," Cancer and Metastasis Reviews, vol. 31, no. 3-4, pp. 469-478, 2012.

[26] D. Sarrió, S. M. Rodriguez-Pinilla, D. Hardisson, A. Cano, G. Moreno-Bueno, and J. Palacios, "Epithelial-mesenchymal transition in breast cancer relates to the basal-like phenotype," Cancer Research, vol. 68, no. 4, pp. 989-997, 2008.

[27] A. J. Trimboli, K. Fukino, A. de Bruin et al., "Direct evidence for epithelial-mesenchymal transitions in breast cancer," Cancer Research, vol. 68, no. 3, pp. 937-945, 2008.

[28] M. Yu, A. Bardia, B. S. Wittner et al., "Circulating breast tumor cells exhibit dynamic changes in epithelial and mesenchymal composition," Science, vol. 339, no. 6119, pp. 580-584, 2013.

[29] D. Entenberg, D. Kedrin, J. Wyckoff, E. Sahai, J. Condeelis, and J. E. Segall, "Imaging tumor cell movement in vivo," in Current Protocols in Cell Biology, chapter 19, unit 19.7, 2013.

[30] Y. Kang and K. Pantel, "Tumor cell dissemination: emerging biological insights from animal models and cancer patients," Cancer Cell, vol. 23, no. 5, pp. 573-581, 2013.

[31] M. J. Blanco, G. Moreno-Bueno, D. Sarrio et al., "Correlation of Snail expression with histological grade and lymph node status in breast carcinomas," Oncogene, vol. 21, no. 20, pp. 3241-3246, 2002.

[32] C. Côme, F. Magnino, F. Bibeau et al., "Snail and slug play distinct roles during breast carcinoma progression," Clinical Cancer Research, vol. 12, no. 18, pp. 5395-5402, 2006.

[33] S. Elloul, M. B. Elstrand, J. M. Nesland et al., "Snail, slug, and smad-interacting protein 1 as novel parameters of disease aggressiveness in metastatic ovarian and breast carcinoma," Cancer, vol. 103, no. 8, pp. 1631-1643, 2005.

[34] T. A. Martin, A. Goyal, G. Watkins, and W. G. Jiang, "Expression of the transcription factors snail, slug, and twist and their 
clinical significance in human breast cancer," Annals of Surgical Oncology, vol. 12, no. 6, pp. 488-496, 2005.

[35] S. Elloul, I. Silins, C. G. Tropé, A. Benshushan, B. Davidson, and R. Reich, "Expression of E-cadherin transcriptional regulators in ovarian carcinoma," Virchows Archiv, vol. 449, no. 5, pp. 520$528,2006$.

[36] T. Imai, A. Horiuchi, C. Wang et al., "Hypoxia attenuates the expression of E-cadherin via up-regulation of SNAIL in ovarian carcinoma cells," The American Journal of Pathology, vol. 163, no. 4, pp. 1437-1447, 2003.

[37] M.-Y. Cai, R.-Z. Luo, J.-W. Chen et al., “Overexpression of ZEB2 in peritumoral liver tissue correlates with favorable survival after curative resection of hepatocellular carcinoma," PLoS ONE, vol. 7, no. 2, Article ID e32838, 2012.

[38] T. Li, Y. Zhu, W. Ren et al., "High co-expression of vascular endothelial growth factor receptor-1 and Snail is associated with poor prognosis after curative resection of hepatocellular carcinoma," Medical Oncology, vol. 29, no. 4, pp. 2750-2761, 2012.

[39] A. Miyoshi, Y. Kitajima, S. Kido et al., "Snail accelerates cancer invasion by upregulating MMP expression and is associated with poor prognosis of hepatocellular carcinoma," British Journal of Cancer, vol. 92, no. 2, pp. 252-258, 2005.

[40] K. Sugimachi, S. Tanaka, T. Kameyama et al., "Transcriptional repressor snail and progression of human hepatocellular carcinoma," Clinical Cancer Research, vol. 9, no. 7, pp. 2657-2664, 2003.

[41] C. Kahlert, S. Lahes, P. Radhakrishnan et al., "Overexpression of ZEB2 at the invasion front of colorectal cancer is an independent prognostic marker and regulates tumor invasion in vitro," Clinical Cancer Research, vol. 17, no. 24, pp. 7654-7663, 2011.

[42] C. Peña, J. M. Garciá, J. Silva et al., "E-cadherin and vitamin $\mathrm{D}$ receptor regulation by SNAIL and ZEB1 in colon cancer: clinicopathological correlations," Human Molecular Genetics, vol. 14, no. 22, pp. 3361-3370, 2005.

[43] H. Wallerand, G. Robert, G. Pasticier et al., "The epithelialmesenchymal transition-inducing factor TWIST is an attractive target in advanced and/or metastatic bladder and prostate cancers," Urologic Oncology, vol. 28, no. 5, pp. 473-479, 2010.

[44] S. D. da Silva, M. A. Alaoui-Jamali, F. A. Soares et al., "TWIST1 is a molecular marker for a poor prognosis in oral cancer and represents a potential therapeutic target," Cancer, vol. 120, no. 3 , pp. 352-362, 2014.

[45] S. Hosono, H. Kajiyama, M. Terauchi et al., "Expression of Twist increases the risk for recurrence and for poor survival in epithelial ovarian carcinoma patients," British Journal of Cancer, vol. 96, no. 2, pp. 314-320, 2007.

[46] H. Kajiyama, S. Hosono, M. Terauchi et al., “Twist expression predicts poor clinical outcome of patients with clear cell carcinoma of the ovary," Oncology, vol. 71, no. 5-6, pp. 394-401, 2007.

[47] K. Shibata, H. Kajiyama, K. Ino et al., "Twist expression in patients with cervical cancer is associated with poor disease outcome," Annals of Oncology, vol. 19, no. 1, pp. 81-85, 2008.

[48] A. Satelli and S. Li, "Vimentin in cancer and its potential as a molecular target for cancer therapy," Cellular and Molecular Life Sciences, vol. 68, no. 18, pp. 3033-3046, 2011.

[49] P. C. Black, G. A. Brown, T. Inamoto et al., "Sensitivity to epidermal growth factor receptor inhibitor requires Ecadherin expression in urothelial carcinoma cells," Clinical Cancer Research, vol. 14, no. 5, pp. 1478-1486, 2008.
[50] M. J. Kwon, J. H. Kwon, E. S. Nam et al., “TWIST1 promoter methylation is associated with prognosis in tonsillar squamous cell carcinoma," Human Pathology, vol. 44, no. 9, pp. 1722-1729, 2013.

[51] J. Kim, S. J. Hong, J. Y. Park et al., "Epithelial - mesenchymal transition gene signature to predict clinical outcome of hepatocellular carcinoma," Cancer Science, vol. 101, no. 6, pp. 1521-1528, 2010.

[52] Y. Niwa, S. Yamada, M. Koike et al., "Epithelial to mesenchymal transition correlates with tumor budding and predicts prognosis in esophageal squamous cell carcinoma," Journal of Surgical Oncology, vol. 110, no. 6, pp. 764-769, 2014.

[53] S. Yamada, N. Okumura, L. Wei et al., "Epithelial to mesenchymal transition is associated with shorter disease-free survival in hepatocellular carcinoma," Annals of Surgical Oncology, vol. 21, no. 12, pp. 3882-3890, 2014.

[54] X. Zhai, H. Zhu, W. Wang, S. Zhang, Y. Zhang, and G. Mao, "Abnormal expression of EMT-related proteins, S100A4, vimentin and E-cadherin, is correlated with clinicopathological features and prognosis in HCC," Medical Oncology, vol. 31, no. 6, article 970, 2014.

[55] J. Zhao, D. Dong, L. Sun, G. Zhang, and L. Sun, "Prognostic significance of the epithelial-tomesenchymal transition markers e-cadherin, vimentin and twist in bladder cancer," International Brazilian Journal of Urology, vol. 40, no. 2, pp. 179-189, 2014.

[56] T. Brabletz, A. Jung, S. Spaderna, F. Hlubek, and T. Kirchner, "Migrating cancer stem cells-an integrated concept of malignant tumour progression," Nature Reviews Cancer, vol. 5, no. 9, pp. 744-749, 2005.

[57] G. Ghiaur, J. Gerber, and R. J. Jones, "Concise review: cancer stem cells and minimal residual disease," Stem Cells, vol. 30, no. 1, pp. 89-93, 2012.

[58] J. Pasquier and A. Rafii, "Role of the microenvironment in ovarian cancer stem cell maintenance," BioMed Research International, vol. 2013, Article ID 630782, 10 pages, 2013.

[59] W. Luo, S. Li, B. Peng, Y. Ye, X. Deng, and K. Yao, "Embryonic stem cells markers SOX2, OCT4 and Nanog expression and their correlations with epithelial-mesenchymal transition in nasopharyngeal carcinoma," PLoS ONE, vol. 8, no. 2, Article ID e56324, 2013.

[60] H. S. Ryu, D. J. Park, H. H. Kim, W. H. Kim, and H. S. Lee, "Combination of epithelial-mesenchymal transition and cancer stem cell-like phenotypes has independent prognostic value in gastric cancer," Human Pathology, vol. 43, no. 4, pp. 520-528, 2012.

[61] H. S. Ryu, J.-H. Chung, K. Lee et al., "Overexpression of epithelial-mesenchymal transition-related markers according to cell dedifferentiation: clinical implications as an independent predictor of poor prognosis in cholangiocarcinoma," Human Pathology, vol. 43, no. 12, pp. 2360-2370, 2012.

[62] Y. Wang, S. Kim, and I. M. Kim, "Regulation of metastasis by microRNAs in ovarian cancer," Frontiers in Oncology, vol. 4, article 143, 2014.

[63] S. F. Tavazoie, C. Alarcón, T. Oskarsson et al., "Endogenous human microRNAs that suppress breast cancer metastasis," Nature, vol. 451, no. 7175, pp. 147-152, 2008.

[64] X. Fu, Y. Han, Y. Wu et al., "Prognostic role of microRNA-21 in various carcinomas: a systematic review and meta-analysis," European Journal of Clinical Investigation, vol. 41, no. 11, pp. 1245-1253, 2011. 
[65] A. Rafii, C. Touboul, H. Al Thani, K. Suhre, and J. A. Malek, "Where cancer genomics should go next: a clinician's perspective," Human Molecular Genetics, vol. 23, no. R1, pp. R69-R75, 2014.

[66] U. H. Frixen, J. Behrens, M. Sachs et al., "E-cadherin-mediated cell-cell adhesion prevents invasiveness of human carcinoma cells," The Journal of Cell Biology, vol. 113, no. 1, pp. 173-185, 1991.

[67] J. Luo, D. M. Lubaroff, and M. J. C. Hendrix, "Suppression of prostate cancer invasive potential and matrix metalloproteinase activity by E-cadherin transfection," Cancer Research, vol. 59, no. 15 , pp. 3552-3556, 1999.

[68] S. E. Witta, R. M. Gemmill, F. R. Hirsch et al., "Restoring Ecadherin expression increases sensitivity to epidermal growth factor receptor inhibitors in lung cancer cell lines," Cancer Research, vol. 66, no. 2, pp. 944-950, 2006.

[69] A. S. T. Wong and B. M. Gumbiner, "Adhesion-independent mechanism for suppression of tumor cell invasion by Ecadherin," The Journal of Cell Biology, vol. 161, no. 6, pp. 11911203, 2003.

[70] M. Yanagisawa and P. Z. Anastasiadis, "p120 catenin is essential for mesenchymal cadherin-mediated regulation of cell motility and invasiveness," Journal of Cell Biology, vol. 174, no. 7, pp. 1087-1096, 2006.

[71] P. B. Gupta, T. T. Onder, G. Jiang et al., "Identification of selective inhibitors of cancer stem cells by high-throughput screening," Cell, vol. 138, no. 4, pp. 645-659, 2009.

[72] B. Zhai, H.-X. Yan, S.-Q. Liu, L. Chen, M.-C. Wu, and H.-Y. Wang, "Reduced expression of E-cadherin/catenin complex in hepatocellular carcinomas," World Journal of Gastroenterology, vol. 14, no. 37, pp. 5665-5673, 2008.

[73] Z.-Q. Ling, P. Li, M.-H. Ge et al., "Hypermethylation-modulated down-regulation of $\mathrm{CDH} 1$ expression contributes to the progression of esophageal cancer," International Journal of Molecular Medicine, vol. 27, no. 5, pp. 625-635, 2011.

[74] I. Molina-Ortiz, R. A. Bartolomé, P. Hernández-Varas, G. P. Colo, and J. Teixidó, "Overexpression of E-cadherin on melanoma cells inhibits chemokine-promoted invasion involving p190RhoGAP/p120ctn-dependent inactivation of RhoA," The Journal of Biological Chemistry, vol. 284, no. 22, pp. 1514715157, 2009.

[75] G. Berx and F. Van Roy, "The E-cadherin/catenin complex: an important gatekeeper in breast cancer tumorigenesis and malignant progression," Breast Cancer Research, vol. 3, no. 5, pp. 289-293, 2001.

[76] K. Strumane, G. Berx, and F. van Roy, "Cadherins in Cancer," in Cell Adhesion, vol. 165 of Handbook of Experimental Pharmacology, pp. 69-103, Springer, Berlin, Germany, 2004.

[77] J. K. Field, "Oncogenes and tumour-suppressor genes in squamous cell carcinoma of the head and neck," European Journal of Cancer Part B: Oral Oncology, vol. 28, no. 1, pp. 67-76, 1992.

[78] B. Ruggeri, J. Caamano, T. J. Slaga, C. J. Conti, W. J. Nelson, and A. J. P. Klein-Szanto, "Alterations in the expression of uvomorulin and $\mathrm{Na}+\mathrm{K}(+)$-adenosine triphosphatase during mouse skin tumor progression," The American Journal of Pathology, vol. 140, no. 5, pp. 1179-1185, 1992.

[79] M. Köbel, D. Turbin, S. E. Kalloger, D. Gao, D. G. Huntsman, and C. B. Gilks, "Biomarker expression in pelvic high-grade serous carcinoma: comparison of ovarian and omental sites," International Journal of Gynecological Pathology, vol. 30, no. 4, pp. 366-371, 2011.

[80] A. S. Wong, S. L. Maines-Bandiera, B. Rosen et al., "Constitutive and conditional cadherin expression in cultured human ovarian surface epithelium: influence of family history of ovarian cancer," International Journal of Cancer, vol. 81, no. 2, pp. 180188, 1999.

[81] F. J. Rodriguez, L. J. Lewis-Tuffin, and P. Z. Anastasiadis, "E-cadherin's dark side: possible role in tumor progression," Biochimica et Biophysica Acta, vol. 1826, no. 1, pp. 23-31, 2012.

[82] T. Celià-Terrassa, Ó. Meca-Cortés, F. Mateo et al., "Epithelialmesenchymal transition can suppress major attributes of human epithelial tumor-initiating cells," The Journal of Clinical Investigation, vol. 122, no. 5, pp. 1849-1868, 2012.

[83] A. Gheldof and G. Berx, "Cadherins and epithelial-to-mesenchymal transition," Progress in Molecular Biology and Translational Science, vol. 116, pp. 317-336, 2013.

[84] M. J. Wheelock, Y. Shintani, M. Maeda, Y. Fukumoto, and K. R. Johnson, "Cadherin switching," Journal of Cell Science, vol. 121, no. 6, pp. 727-735, 2008.

[85] Y. Shintani, Y. Fukumoto, N. Chaika et al., "ADH-1 suppresses $\mathrm{N}$-cadherin-dependent pancreatic cancer progression," International Journal of Cancer, vol. 122, no. 1, pp. 71-77, 2008.

[86] W.-W. Chang, F.-W. Hu, C.-C. Yu et al., "Quercetin in elimination of tumor initiating stem-like and mesenchymal transformation property in head and neck cancer," Head and Neck, vol. 35, no. 3, pp. 413-419, 2013.

[87] N. M. Sadler, B. R. Harris, B. A. Metzger, and J. Kirshner, "Ncadherin impedes proliferation of the multiple myeloma cancer stem cells," The American Journal of Blood Research, vol. 3, no. 4, pp. 271-285, 2013.

[88] G. Lahat, Q.-S. Zhu, K.-L. Huang et al., "Vimentin is a novel anti-cancer therapeutic target; insights from In Vitro and In Vivo mice xenograft studies," PLoS ONE, vol. 5, no. 4, Article ID e10105, 2010.

[89] R. P. Singh, K. Raina, G. Sharma, and R. Agarwal, "Silibinin inhibits established prostate tumor growth, progression, invasion, and metastasis and suppresses tumor angiogenesis and epithelial-mesenchymal transition in transgenic adenocarcinoma of the mouse prostate model mice," Clinical Cancer Research, vol. 14, no. 23, pp. 7773-7780, 2008.

[90] K. J. Wu, J. Zeng, G. D. Zhu et al., "Silibinin inhibits prostate cancer invasion, motility and migration by suppressing vimentin and MMP-2 expression," Acta Pharmacologica Sinica, vol. 30, no. 8, pp. 1162-1168, 2009.

[91] T.-T. Dong, H.-M. Zhou, L.-L. Wang, B. Feng, B. Lv, and M.-H. Zheng, 'Salinomycin selectively targets 'CD133+' cell subpopulations and decreases malignant traits in colorectal cancer lines," Annals of Surgical Oncology, vol. 18, no. 6, pp. 1797-1804, 2011.

[92] Y. Teng and X. Li, “The roles of HLH transcription factors in epithelial mesenchymal transition and multiple molecular mechanisms," Clinical and Experimental Metastasis, vol. 31, no. 3, pp. 367-377, 2014.

[93] S.-P. Han, J.-H. Kim, M.-E. Han et al., "SNAI1 is involved in the proliferation and migration of glioblastoma cells," Cellular and Molecular Neurobiology, vol. 31, no. 3, pp. 489-496, 2011.

[94] S. A. Mikheeva, A. M. Mikheev, A. Petit et al., "TWIST1 promotes invasion through mesenchymal change in human glioblastoma," Molecular Cancer, vol. 9, article 194, 2010.

[95] M. Xia, M. Hu, J. Wang et al., "Identification of the role of Smad interacting protein 1 (SIP1) in glioma," Journal of NeuroOncology, vol. 97, no. 2, pp. 225-232, 2010.

[96] M.-T. Chung, H.-C. Lai, H.-K. Sytwu et al., "SFRP1 and SFRP2 suppress the transformation and invasion abilities of 
cervical cancer cells through Wnt signal pathway," Gynecologic Oncology, vol. 112, no. 3, pp. 646-653, 2009.

[97] Y.-R. Na, S.-H. Seok, D.-J. Kim et al., "Bone morphogenetic protein 7 induces mesenchymal-to-epithelial transition in melanoma cells, leading to inhibition of metastasis," Cancer Science, vol. 100, no. 11, pp. 2218-2225, 2009.

[98] T. Arumugam, V. Ramachandran, K. F. Fournier et al., "Epithelial to mesenchymal transition contributes to drug resistance in pancreatic cancer," Cancer Research, vol. 69, no. 14, pp. 5820$5828,2009$.

[99] W. Zhuo, Y. Wang, X. Zhuo, Y. Zhang, X. Ao, and Z. Chen, "Knockdown of Snail, a novel zinc finger transcription factor, via RNA interference increases A549 cell sensitivity to cisplatin via JNK/mitochondrial pathway," Lung Cancer, vol. 62, no. 1, pp. 8-14, 2008.

[100] W.-L. Zhuo, Y. Wang, X.-L. Zhuo, Y.-S. Zhang, and Z.-T. Chen, "Short interfering RNA directed against TWIST, a novel zinc finger transcription factor, increases A549 cell sensitivity to cisplatin via MAPK/mitochondrial pathway," Biochemical and Biophysical Research Communications, vol. 369, no. 4, pp. 10981102, 2008.

[101] R. K. Srivastava, S.-N. Tang, W. Zhu, D. Meeker, and S. Shankar, "Sulforaphane synergizes with quercetin to inhibit self-renewal capacity of pancreatic cancer stem cells," Frontiers in Bioscience, Elite, vol. 3, no. 2, pp. 515-528, 2011.

[102] H.-C. Pai, L.-H. Chang, C.-Y. Peng et al., "Moscatilin inhibits migration and metastasis of human breast cancer MDA-MB231 cells through inhibition of Akt and Twist signaling pathway," Journal of Molecular Medicine, vol. 91, no. 3, pp. 347-356, 2013.

[103] H.-Y. Hsu, T.-Y. Lin, P.-A. Hwang et al., "Fucoidan induces changes in the epithelial to mesenchymal transition and decreases metastasis by enhancing ubiquitin-dependent TGFbeta receptor degradation in breast cancer," Carcinogenesis, vol. 34, no. 4, pp. 874-884, 2013.

[104] J. K. Myung, S. A. Choi, S. K. Kim, K. C. Wang, and S. H. Park, "Snail plays an oncogenic role in glioblastoma by promoting epithelial mesenchymal transition," International Journal of Clinical and Experimental Pathology, vol. 7, no. 5, pp. 1977-1987, 2014.

[105] A. K. Reka, R. Kuick, H. Kurapati, T. J. Standiford, G. S. Omenn, and V. G. Keshamouni, "Identifying inhibitors of epithelialmesenchymal transition by connectivity map-based systems approach," Journal of Thoracic Oncology, vol. 6, no. 11, pp. 17841792, 2011.

[106] K.-N. Chua, W.-J. Sim, V. Racine, S.-Y. Lee, B. C. Goh, and J. P. Thiery, "A cell-based small molecule screening method for identifying inhibitors of epithelial-mesenchymal transition in carcinoma," PLoS ONE, vol. 7, no. 3, Article ID e33183, 2012.

[107] H. You, W. Ding, H. Dang, Y. Jiang, and C. B. Rountree, "cMet represents a potential therapeutic target for personalized treatment in hepatocellular carcinoma," Hepatology, vol. 54, no. 3, pp. 879-889, 2011.

[108] O. O. Ogunwobi and C. Liu, "Hepatocyte growth factor upregulation promotes carcinogenesis and epithelial-mesenchymal transition in hepatocellular carcinoma via Akt and COX-2 pathways," Clinical and Experimental Metastasis, vol. 28, no. 8, pp. 721-731, 2011.

[109] Y. Guo, J. Xie, E. Rubin et al., "Frzb, a secreted Wnt antagonist, decreases growth and invasiveness of fibrosarcoma cells associated with inhibition of Met signaling," Cancer Research, vol. 68, no. 9, pp. 3350-3360, 2008.
[110] H. Ikushima and K. Miyazono, "TGFB 2 signalling: a complex web in cancer progression," Nature Reviews Cancer, vol. 10, no. 6, pp. 415-424, 2010.

[111] Y. Katsuno, S. Lamouille, and R. Derynck, "TGF- $\beta$ signaling and epithelial-mesenchymal transition in cancer progression," Current Opinion in Oncology, vol. 25, no. 1, pp. 76-84, 2013.

[112] J. Xu, S. Lamouille, and R. Derynck, “TGF-B-induced epithelial to mesenchymal transition," Cell Research, vol. 19, no. 2, pp. 156172, 2009.

[113] H. Peng, O. A. Carretero, N. Vuljaj et al., "Angiotensin-converting enzyme inhibitors: a new mechanism of action," Circulation, vol. 112, no. 16, pp. 2436-2445, 2005.

[114] G. Subramanian, R. E. Schwarz, L. Higgins et al., “Targeting endogenous transforming growth factor $\beta$ receptor signaling in Smad4-deficient human pancreatic carcinoma cells inhibits their invasive phenotype," Cancer Research, vol. 64, no. 15, pp. 5200-5211, 2004.

[115] C. Y. Park, D. K. Kim, and Y. Y. Sheen, "EW-7203, a novel small molecule inhibitor of transforming growth factor-beta (TGFbeta) type I receptor/activin receptor-like kinase-5, blocks TGF-betal-mediated epithelial-to-mesenchymal transition in mammary epithelial cells," Cancer Science, vol. 102, no. 10, pp. 1889-1896, 2011.

[116] C.-Y. Park, J.-Y. Son, C. H. Jin, J.-S. Nam, D.-K. Kim, and Y. Y. Sheen, "EW-7195, a novel inhibitor of ALK5 kinase inhibits EMT and breast cancer metastasis to lung," European Journal of Cancer, vol. 47, no. 17, pp. 2642-2653, 2011.

[117] Y. Y. Sheen, M.-J. Kim, S.-A. Park, S.-Y. Park, and J.-S. Nam, "Targeting the transforming growth factor- $\beta$ signaling in cancer therapy," Biomolecules and Therapeutics, vol. 21, no. 5, pp. 323331, 2013.

[118] K. Duangkumpha, A. Techasen, W. Loilome et al., "BMP-7 blocks the effects of TGF- $\beta$-induced EMT in cholangiocarcinoma," Tumor Biology, vol. 35, no. 10, pp. 9667-9676, 2014.

[119] A. R. Tan, G. Alexe, and M. Reiss, "Transforming growth factor- $\beta$ signaling: emerging stem cell target in metastatic breast cancer?" Breast Cancer Research and Treatment, vol. 115, no. 3, pp. 453-495, 2009.

[120] L. Bueno, D. P. de Alwis, C. Pitou et al., "Semi-mechanistic modelling of the tumour growth inhibitory effects of LY2157299, a new type I receptor TGF-beta kinase antagonist, in mice," European Journal of Cancer, vol. 44, no. 1, pp. 142-150, 2008.

[121] J. Rodon, R. Dienstmann, V. Serra, and J. Tabernero, "Development of PI3K inhibitors: lessons learned from early clinical trials," Nature Reviews Clinical Oncology, vol. 10, no. 3, pp. 143153, 2013.

[122] F. Cappuzzo, T. Ciuleanu, L. Stelmakh et al., "Erlotinib as maintenance treatment in advanced non-small-cell lung cancer: a multicentre, randomised, placebo-controlled phase 3 study," The Lancet Oncology, vol. 11, no. 6, pp. 521-529, 2010.

[123] F. A. Shepherd, J. R. Pereira, T. Ciuleanu et al., "Erlotinib in previously treated non-small-cell lung cancer," The New England Journal of Medicine, vol. 353, no. 2, pp. 123-132, 2005.

[124] S. E. Witta, R. M. Jotte, K. Konduri et al., "Randomized phase II trial of erlotinib with and without entinostat in patients with advanced non-small-cell lung cancer who progressed on prior chemotherapy," Journal of Clinical Oncology, vol. 30, no. 18, pp. 2248-2255, 2012.

[125] J. C. Morris, A. R. Tan, T. E. Olencki et al., "Phase I study of GC1008 (Fresolimumab): a human anti-transforming growth factor-beta (TGF $\beta$ ) monoclonal antibody in patients with 
advanced malignant melanoma or renal cell carcinoma," PLoS ONE, vol. 9, no. 3, Article ID e90353, 2014.

[126] J. A. Malek, E. Mery, Y. A. Mahmoud et al., "Copy number variation analysis of matched ovarian primary tumors and peritoneal metastasis," PLoS ONE, vol. 6, no. 12, Article ID e28561, 2011.

[127] R. Lis, J. Capdet, P. Mirshahi et al., "Oncologic trogocytosis with Hospicells induces the expression of $\mathrm{N}$-cadherin by breast cancer cells," International Journal of Oncology, vol. 37, no. 6, pp. 1453-1461, 2010.

[128] R. Lis, C. Touboul, N. M. Halabi et al., "Mesenchymal cell interaction with ovarian cancer cells induces a background dependent pro-metastatic transcriptomic profile," Journal of Translational Medicine, vol. 12, article 59, 2014.

[129] R. Lis, C. Touboul, C. M. Raynaud et al., "Mesenchymal cell interaction with ovarian cancer cells triggers pro-metastatic properties," PLoS ONE, vol. 7, no. 5, Article ID e38340, 2012.

[130] J. A. Malek, A. Martinez, E. Mery et al., "Gene expression analysis of matched ovarian primary tumors and peritoneal metastasis," Journal of Translational Medicine, vol. 10, no. 1, article 121, 2012.

[131] J. Pasquier, B. S. Guerrouahen, H. Al Thawadi et al., "Preferential transfer of mitochondria from endothelial to cancer cells through tunneling nanotubes modulates chemoresistance," Journal of Translational Medicine, vol. 11, no. 1, article 94, 2013.

[132] J. Pasquier, H. A. Thawadi, P. Ghiabi et al., "Microparticles mediated cross-talk between tumoral and endothelial cells promote the constitution of a pro-metastatic vascular niche through Arf6 up regulation," Cancer Microenvironment, vol. 7, no. 1-2, pp. 41-59, 2014.

[133] C. Touboul, R. Lis, H. Al Farsi et al., "Mesenchymal stem cells enhance ovarian cancer cell infiltration through IL6 secretion in an amniochorionic membrane based 3D model," Journal of Translational Medicine, vol. 11, no. 1, article 28, 2013. 


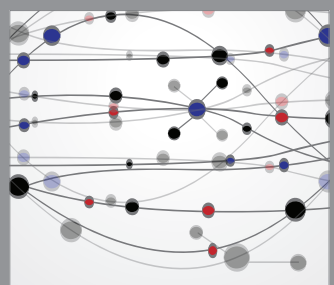

The Scientific World Journal
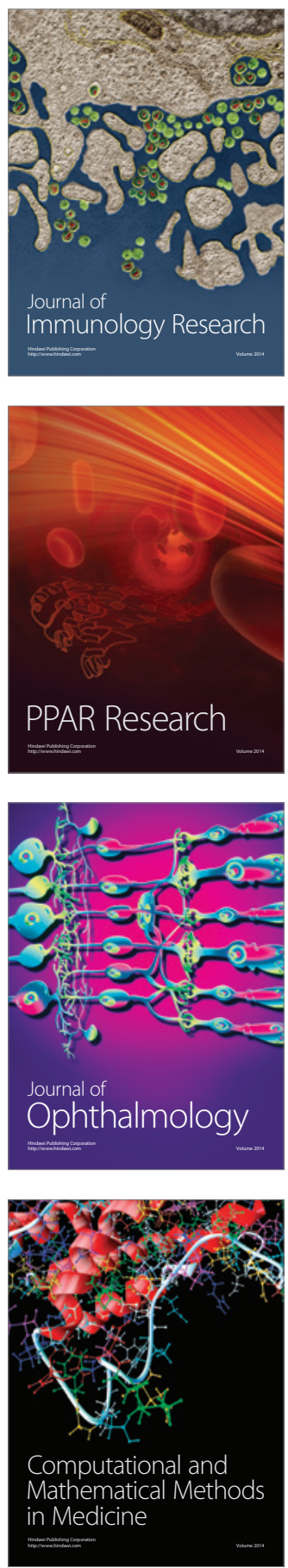

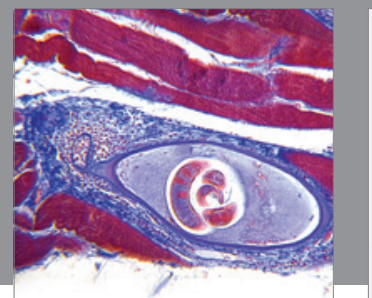

Gastroenterology

Research and Practice
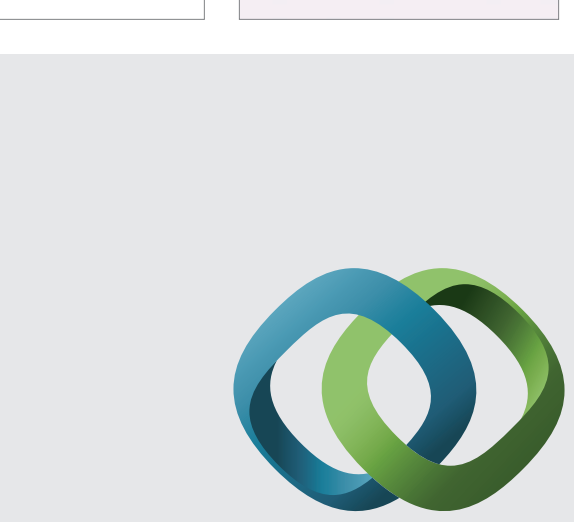

\section{Hindawi}

Submit your manuscripts at

http://www.hindawi.com
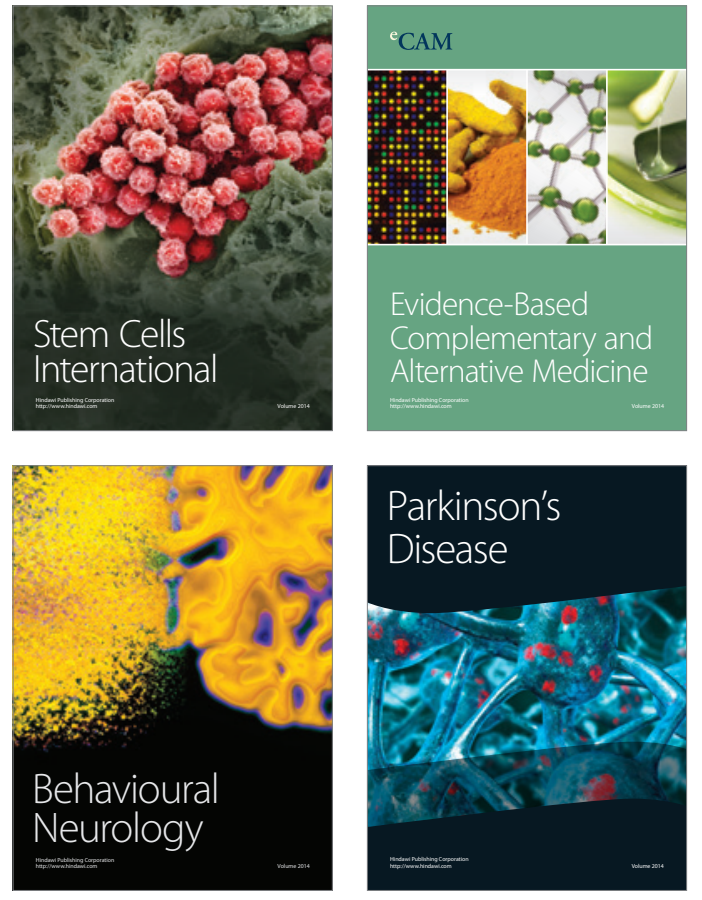
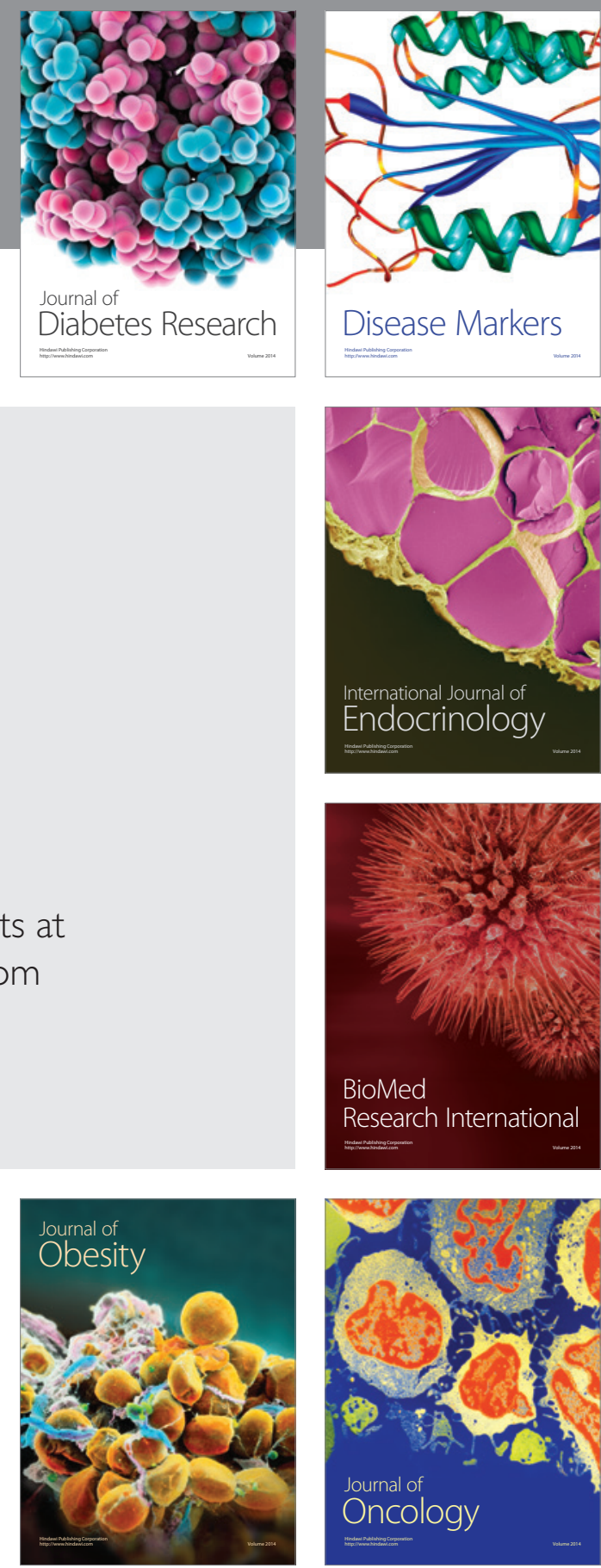

Disease Markers
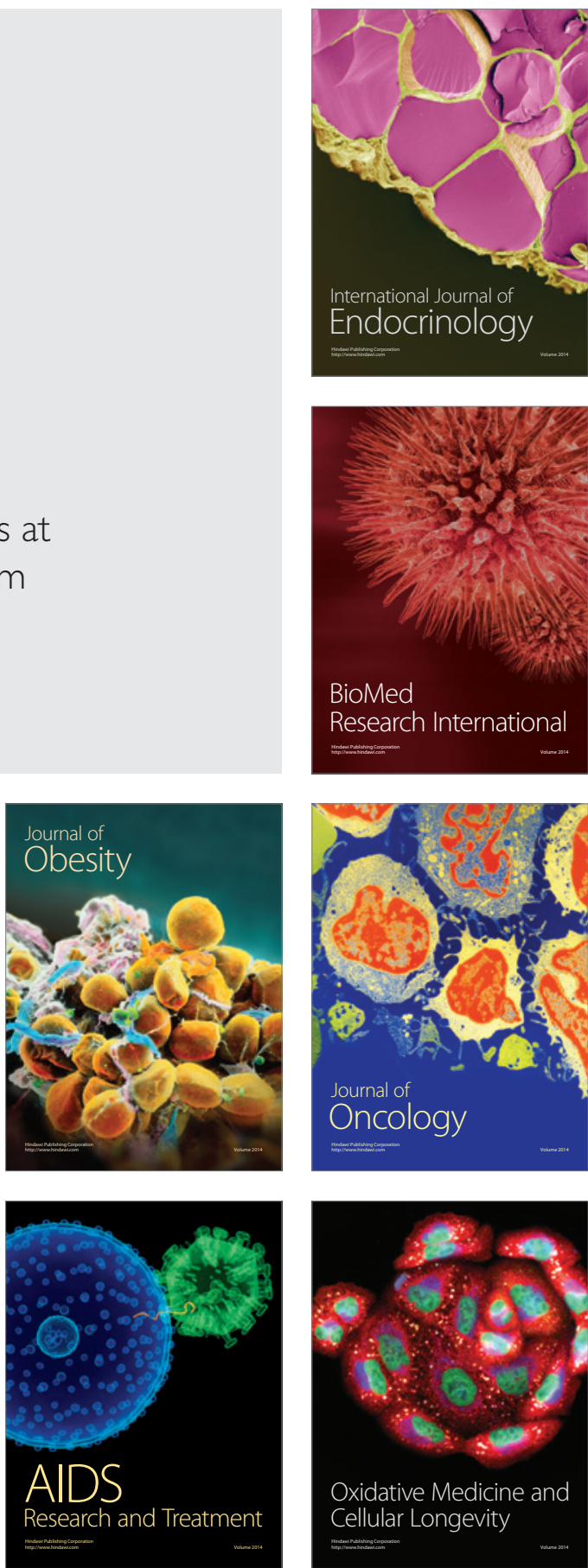